\title{
Increased porosity turns desorption to adsorption for gas bubbles near water-SiO interface
}

\author{
M. Boström, ${ }^{1, *}$ M. Dou, ${ }^{2, \dagger}$ P. Thiyam, ${ }^{2, \dagger}$ D. F. Parsons, ${ }^{3}$ O. I. Malyi, ${ }^{1}$ and C. Persson ${ }^{4,1,2}$ \\ ${ }^{1}$ Centre for Materials Science and Nanotechnology, University of Oslo, P. O. Box 1048, Blindern, NO-0316 Oslo, Norway \\ ${ }^{2}$ Department of Materials Science and Engineering, Royal Institute of Technology, SE-100 44 Stockholm, Sweden \\ ${ }^{3}$ Department of Applied Mathematics, Australian National University, ACT 2601 Canberra, Australia \\ ${ }^{4}$ Department of Physics, University of Oslo, P. O. Box 1048, Blindern, NO-0316 Oslo, Norway
}

(Received 13 October 2014; revised manuscript received 20 November 2014; published 2 February 2015)

\begin{abstract}
We consider theoretically the retarded van der Waals interaction of a small gas bubble in water with a porous $\mathrm{SiO}_{2}$ surface. We predict a possible transition from repulsion to attraction as the surface is made more porous. It highlights that bubbles will interact differently with surface regions with different porosity (i.e., with different optical properties).
\end{abstract}

DOI: 10.1103/PhysRevB.91.075403

PACS number(s): 77.22.Ch, 34.20.Cf, 42.50.Lc, 03.70.+k

\section{INTRODUCTION}

Boundary effects on the electromagnetic fluctuations can produce attraction between a pair of parallel, closely spaced, perfect conductors a distance apart [1-3]. A well-known aspect of the Casimir-Lifshitz force is that, according to theory, it can also be repulsive [3-6]. It has been speculated that this effect could allow quantum levitation of nanoscale devices [6-8]. It was demonstrated by Phan and Viet [9], and by Boström et al. [10], that the force may change sign as the separation is increased. This was known a long time ago from experiments and theoretical calculations studying films on surfaces $[3,11-$ 17], but only a few force measurements of repulsive CasimirLifshitz forces have been reported in the literature [6,18-20]. In the present work, we will study a system where an attractive-repulsive van der Waals force transition occurs in a model system that may potentially provide some insights for an industrially relevant system concerning, in particular, the carbon sequestration and methane recovery technology. Surfaces generated by hydraulic fracturing are mostly mineral surfaces such as shales, silica, etc. We present the result from simple model calculations in order to study trends in the adsorption of bubbles near $\mathrm{SiO}_{2}$ with increasing porosity. The pore structure of shale below the sea floor is not well known, and we cannot draw any definite conclusions for such systems. First, the shale composition varies significantly from place to place. Second, hydrofracturing can induce significant changes to the porosity. Our calculations can be seen as simple models aiming to discuss the trends only. A more well-known system is mesoporous $\mathrm{SiO}_{2}$, where the pore diameters are somewhere between 2 and $50 \mathrm{~nm}[21,22]$. For that kind of system more refined models for the effective dielectric functions may be needed, especially for modeling surfaces separated by only a few nanometers. At this scale, it is also more than likely that a porous material has corrugations that can influence the interaction [23-25]. Bubbles can often be attached to a surface due to chemical or physical inhomogeneities on the surface with a binding energy that can be much larger than the van der Waals energy. A well-known example is

\footnotetext{
*Mathias.Bostrom@smn.uio.no

†maofeng.dou@mse.kth.se

${ }^{\ddagger}$ thiyam@kth.se
}

electrochemical electrodes covered with a gas. The bubbles bind to the electrode, although these gas bubbles that are separated by a liquid gap from a metal are expected to experience a van der Waals repulsion. This coalescence is a serious technological issue. However, Tabor et al. [26] could observe repulsion in such a three-phase system in their measurement of nonequilibrium hydrodynamic interactions between bubbles and solid surfaces in water. The strength of this repulsive van der Waals interaction is controlled only by the choice of solid. In this paper, we theoretically demonstrate the possibility of manipulating the van der Waals interactions by inducing more pores and thereby inducing different optical properties on the silica surface.

During the past years, a number of studies have been focused on the understanding of shale systems and their behaviors under hydraulic fracturing conditions [27-32]. In particular, the analysis of structural properties of the shales $[27,31,32]$ and the shale- $-\mathrm{CO}_{2} / \mathrm{H}_{2} \mathrm{O}$ interaction $[30,33]$ was performed. These studies showed that no two shales are alike, but many properties of the shales are often determined by inorganic matter (e.g., $\mathrm{SiO}_{2}$ ). It is well known that fracturing the materials produces an increase in crack density [28], affecting not only the gas transport but also the molecular storage mechanisms [30]. For instance, Luhmann et al. showed that a change in shale porosity can induce $\mathrm{CO}_{2}$ trapping in the pores [30]. Recent investigations also indicate that the formation of $\mathrm{CO}_{2}$ bubbles in water plays a key role in the storage of $\mathrm{CO}_{2}$ in shale systems [33,34] Moreover, $\mathrm{CO}_{2}$ bubbles are found to be highly stable and could provide $\mathrm{CO}_{2}$ trapping for a number of years [33]. Despite the above studies, the physical and chemical picture of the $\mathrm{CO}_{2}$-shale interaction, and especially the role of system porosity in the interaction, are not yet well studied. Because of this, we aim to show that fracking that induces more pores in the shale potentially can change the way gas bubbles (e.g., $\mathrm{CO}_{2}$ bubbles) in water interact with $\mathrm{SiO}_{2}$ surfaces. In order to highlight the main ideas, we consider bubbles at least a few hundred nanometers in size so that we can use the Derjaguin approximation to estimate well the Casimir force between such a bubble in water with a porous $\mathrm{SiO}_{2}$ surface. We have performed calculations for $0 \%-50 \%$ porosity (fraction of pores) as it is not physically possible to study systems with very large porosity. At higher porosity the pores start to overlap and will eventually be filled with water. The main results we obtain are as follows: For 
$0 \%, 10 \%, 20 \%$, and $30 \%$ pores there is a transition from attraction to repulsion as the surface separation goes under $354,239,126$, and $20 \AA$. At higher porosity there is attraction for all surface separations. These are results obtained from the volume averaging theory (VAT) model for gas pores. The results are only slightly modified when, for instance, the Lorentz-Lorenz effective dielectric functions are used. We can estimate with confidence only what occurs at low porosity, i.e., relatively thick water films, which means we focus on the $0 \%-20 \%$ porosity range where the separation between the surfaces is more than $100 \AA$. This enables us to study trends. For higher porosity the film thickness is very small and the results are highly speculative since the molecular size and surface roughness will play an important role [23-25]. We can still infer that by inducing more pores, a transition from desorption to adsorption of bubbles is predicted near a porous $\mathrm{SiO}_{2}$ in water. This is in line with the work of Lifshitz et al. and others [3-8], which predicts changes in attraction to repulsion depending on the dielectric functions in the three-layer system. Gas bubbles that could include $\mathrm{CO}_{2}$ molecules may bind by physisorption to a porous $\mathrm{SiO}_{2}$ surface. We investigate how $\mathrm{CO}_{2}$ molecules that escape from the gas bubble into the region between the bubble and the porous silica surface bind by a van der Waals interaction to the porous surface. The aim of much research, far beyond the present work, is to understand how a $\mathrm{CO}_{2}$ bubble sticks to a porous surface, and to have $\mathrm{CO}_{2}$ molecules enter into a pore. Here we have the more modest aim to understand the Casimir-Lifshitz interaction between a large bubble in water with a model porous $\mathrm{SiO}_{2}$ surface. We first discuss the dielectric functions and theory used. A few numerical results will be presented and we end with some conclusions.

\section{MODELING OF THE DIELECTRIC FUNCTIONS}

The dielectric function of water [35] at room temperature ( $T=300 \mathrm{~K}$ ) was based on the extensive experimental data found in Ref. [36]. At separations below a few $\mathrm{nm}$ the description of the optical response of water may be modified compared to that of the bulk liquid. We note, however, that for many materials it turns out that they approach the bulk limit for very thin films (e.g., a gold film with ten atomic layers has a dielectric function close to that of bulk gold [37]). Here the main focus is on water films $100 \AA$ or larger, although we stretch the limits and also estimate what trends follow for thinner films.

The complex dielectric function for $\mathrm{SiO}_{2}$ (see Fig. 1) was determined by employing a first-principles approach within the density functional theory [7]. The electronic structure, neglecting electron-phonon coupling, was obtained from the partial self-consistent $G W_{0}$ method based on the local density approximation, where the Green's functions are updated iteratively whereas the screened potential $W$ is fixed $[38,39]$. The imaginary part of the dielectric function was calculated in the long wavelength limit from the joint density of states and the optical momentum matrix. Since the dielectric function in polar materials can depend strongly on the phonon contribution at low frequencies, we modeled the electron-phonon coupling by adding a deltalike contribution to the imaginary part of the dielectric function at the transverse phonon frequency [7]. The mesoporous material consists of a host medium $\left(\mathrm{SiO}_{2}\right)$

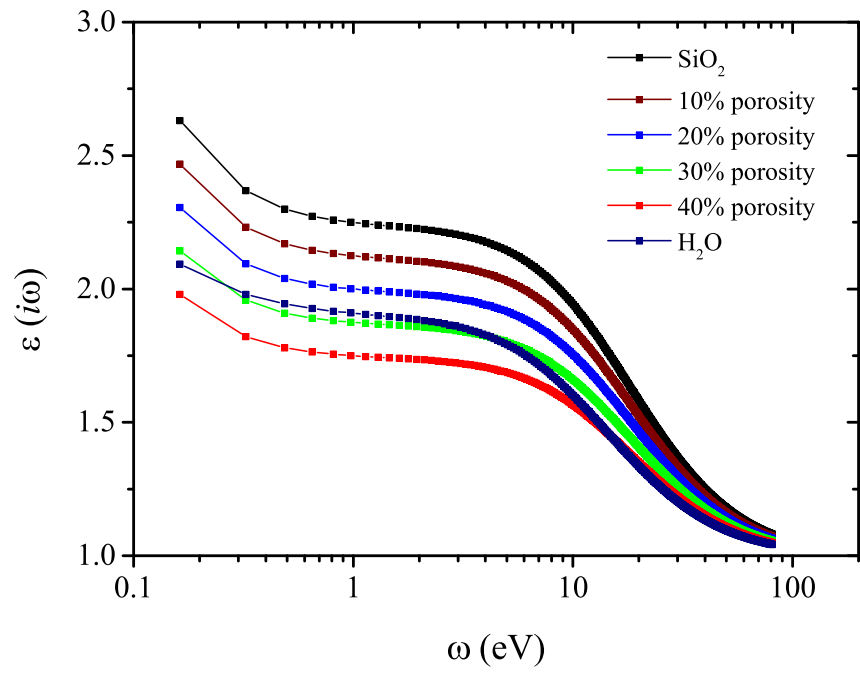

FIG. 1. (Color online) The dielectric functions at imaginary Matsubara frequencies from $n \geqslant 1$. The values for $n=0$ are 77.98, 3.95, $3.65,3.36,3.06$, and 2.77 for $\mathrm{H}_{2} \mathrm{O}, \mathrm{SiO}_{2}, 10 \%, 20 \%, 30 \%$, and $40 \%$, respectively. The dielectric functions with a finite porosity are for the VAT model with air pores. This is to exemplify one of the four models used in the present work.

and nanopores where its dielectric function can be fine tuned by modifying the pore structure and the amount of pores by surface cracking. To investigate this concept, hexagonal mesoporous silica (MCM-41) can be taken as a typical example. The dielectric function is calculated using the effective medium approximation (EMA), wherein the material is treated as a homogeneous medium. The validity of the EMA approach can be confirmed through comparisons with numerical calculations [40]. For the transverse electric (TE) polarized wave, EMA described by the volume averaging theory (VAT) matches accurately with numerical results, while for the transverse magnetic (TM) polarized light the parallel model [41] gives the best predictions. Mathematically, the effective dielectric function for the VAT models is $\epsilon_{\text {eff }}=$ $(1-\phi) \epsilon_{c}+\phi \epsilon_{d}$, where $\phi$ is the porosity, and $\epsilon_{c}$ and $\epsilon_{d}$ are the dielectric functions of the continuous (silica) and the dispersed (air or water) phases, respectively. There are different nanopores in the $\mathrm{SiO}_{2}$ material and the pores have different sizes and shapes, and therefore we model with an "effective porosity." To study the effects of the specific model used we consider also the Lorentz-Lorenz effective dielectric function model $\left(\left[\left(\epsilon_{\mathrm{eff}}-1\right) /\left(\epsilon_{\mathrm{eff}}+2\right)\right]=\sum_{i} f_{i}\left[\left(\epsilon_{i}-1\right) /\left(\epsilon_{i}+\right.\right.\right.$ $2)]$, where $f_{i}$ and $\epsilon_{i}$ are the fraction and dielectric functions of phase $i$ ) and study both air and water filled pores [41,42]. The macroscopic model is reasonable approximation for pore radii sufficiently larger than the atomic scale. We determined the dielectric functions on the imaginary frequency axis using one version of the Kramers-Kronig dispersion relation [35].

\section{REPULSIVE CASIMIR-LIFSHITZ FORCES}

The Casimir force at temperature $T$ can be calculated if the dielectric functions (for discrete imaginary frequencies, $\left.i \omega_{n}=2 \pi n k_{B} T / \hbar\right)$ are known. Using the Deryaguin approximation [43], the Casimir-Lifshitz force of a planar surface of material 1 (porous silica) with a sphere (radius $R$ ) of material 3 
(gas bubble) across medium 2 (water) results in a summation of imaginary frequency terms $[3,35,43]$ :

$$
F /(2 \pi R)=\sum_{n=0}^{\infty} \prime g\left(\omega_{n}\right) .
$$

Note that positive values of $F$ correspond to repulsive force.

In the retarded treatment, there are contributions from the two mode types, transverse magnetic (TM) and transverse electric (TE), $g\left(\omega_{n}\right)=g^{\mathrm{TM}}\left(\omega_{n}\right)+g^{\mathrm{TE}}\left(\omega_{n}\right)$, where

$$
\begin{aligned}
g^{\mathrm{TM}}\left(\omega_{n}\right)= & \frac{1}{\beta} \int \frac{d^{2} q}{(2 \pi)^{2}} \\
& \times \ln \left\{1-e^{-2 \gamma_{2} d}\left[\frac{\varepsilon_{1}\left(i \omega_{n}\right) \gamma_{2}-\varepsilon_{2}\left(i \omega_{n}\right) \gamma_{1}}{\varepsilon_{1}\left(i \omega_{n}\right) \gamma_{2}+\varepsilon_{2}\left(i \omega_{n}\right) \gamma_{1}}\right]\right. \\
& \left.\times\left[\frac{\varepsilon_{3}\left(i \omega_{n}\right) \gamma_{2}-\varepsilon_{2}\left(i \omega_{n}\right) \gamma_{3}}{\varepsilon_{3}\left(i \omega_{n}\right) \gamma_{2}+\varepsilon_{2}\left(i \omega_{n}\right) \gamma_{3}}\right]\right\} \\
g^{\mathrm{TE}}\left(\omega_{n}\right)= & \frac{1}{\beta} \int \frac{d^{2} q}{(2 \pi)^{2}} \\
& \times \ln \left\{1-e^{-2 \gamma_{2} d}\left[\frac{\gamma_{2}-\gamma_{1}}{\gamma_{2}+\gamma_{1}}\right]\left[\frac{\gamma_{2}-\gamma_{3}}{\gamma_{2}+\gamma_{3}}\right]\right\} \\
\gamma_{i}= & \sqrt{q^{2}-\varepsilon_{i}\left(i \omega_{n}\right)\left(i \omega_{n} / c\right)^{2}} .
\end{aligned}
$$

Frequency intervals where the intervening medium has a dielectric permittivity in between the permittivity of the two objects give a repulsive contribution; other intervals give an attractive contribution. The calculation of the force needs accurate dielectric functions for a wide frequency region for precise predictions. Via tuning the porosity (created by cracking of the surface material), the optical properties can be fine tuned so that there can be repulsion at specific separations.

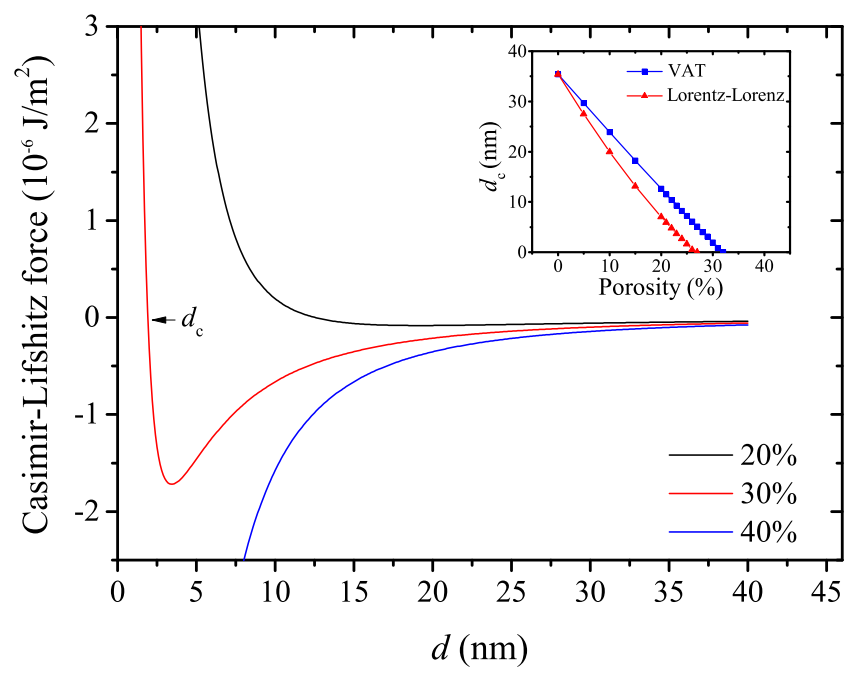

FIG. 2. (Color online) The Casimir-Lifshitz force [normalized with the factor $1 /(2 \pi R)$ ] as a function of the closest distance between the plane and the sphere for different percentages of pores in the $\mathrm{SiO}_{2}$ surface (for a VAT model with air pores). The inset shows the critical distance $d_{c}$ (zero force distance) as a function of porosity (for the VAT model and Lorentz-Lorenz model with air pores).

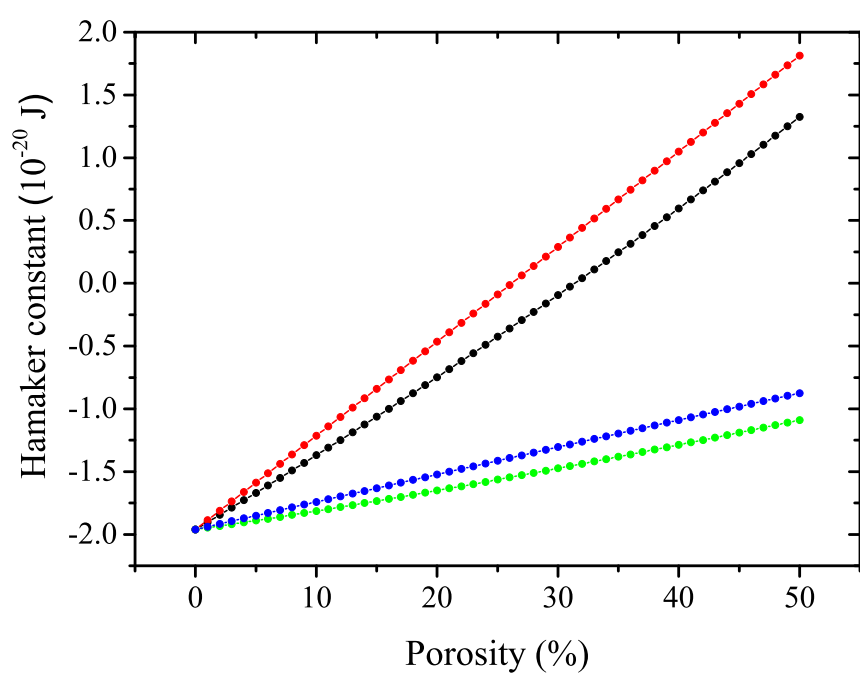

FIG. 3. (Color online) The Hamaker constant vs porosity. Note that values above $30 \%$ porosity start to become unphysical. This is because pores eventually start to overlap, and water will go into the pores at high porosity. The curves correspond to Lorentz-Lorenz air (red curve), VAT air (black curve), Lorentz-Lorenz water (blue curve), and VAT water (green curve) dielectric models.

In Fig. 2 we show the retarded force [normalized with the factor $1 /(2 \pi R)$ ] between a porous $\mathrm{SiO}_{2}$ surface with $20 \%$, $30 \%$, and $40 \%$ porosity interacting with a bubble $\left(\epsilon_{3}=1\right)$ in water. For high porosity, the pores will be filled with water, but we focus on what happens at low porosity. For 0\%-30\% pores there is a transition from attraction to repulsion as the surface separation goes under a critical distance (see the inset in Fig. 2). At more than $30 \%$ pores, there is attraction for all surface separations. Therefore, by inducing more pores, we predict a transition from desorption to adsorption of bubbles near a porous $\mathrm{SiO}_{2}$ in water. The nonretarded (NR) limit can be investigated by letting the velocity of light go to infinity. The Hamaker constant is obtained by taking $A=-12 \pi d^{2} F^{\mathrm{NR}} /(2 \pi R)$. In Fig. 3 we present the Hamaker constant versus pore percentage. These Hamaker constants can be used to calculate the nonretarded force using the Derjaguin approximation with the given relationship between the Hamaker constant and the nonretarded energy between planar surfaces. Here again we see a transition from repulsion to attraction as the air pore percentage increases. For an increasing concentration of water pores, there is reduced repulsion. The VAT and Lorentz-Lorenz models produce similar results.

In this connection we would like to point out that any $\mathrm{CO}_{2}$ molecules that leak from the gas bubble into water will interact via van der Waals interactions with the water-porous $\mathrm{SiO}_{2}$ interface. The excess polarizabilities of $\mathrm{CO}_{2}$ molecules in water at Matsubara frequencies and Gaussian radii for $\mathrm{CO}_{2}$ solved in water were derived as in, for instance, papers by Parsons [44-46]. These quantities can be used to calculate the van der Waals energy between a finite size molecule and an interface [44]. The van der Waals contribution to the binding energy of a $\mathrm{CO}_{2}$ molecule near the porous $\mathrm{SiO}_{2}$-water interface as a function of porosity is shown in Fig. 4. Here we see that as the porosity (average volume of air filled regions) 


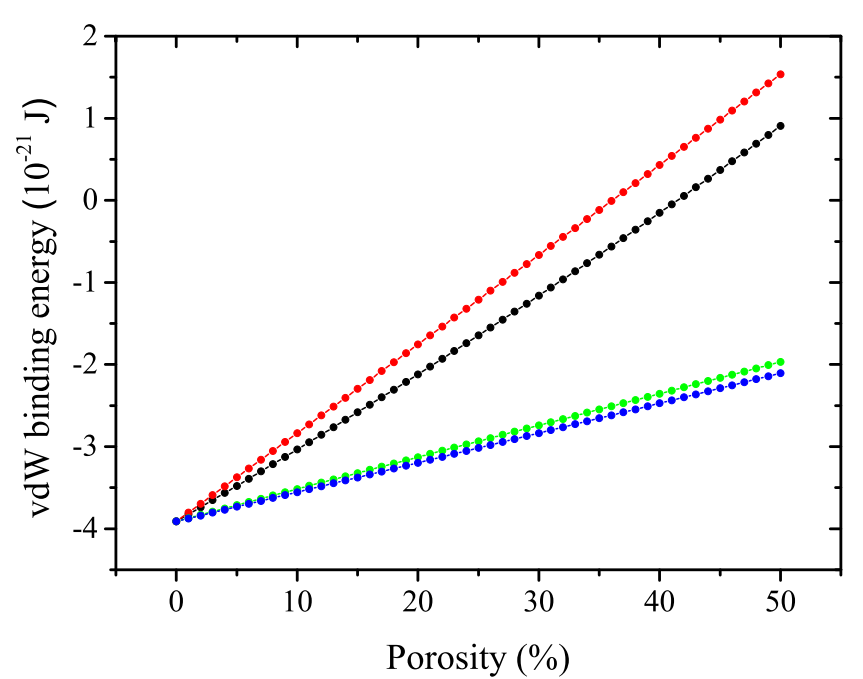

FIG. 4. (Color online) The van der Waals (vdW) binding energy of $\mathrm{CO}_{2}$ near a water-porous $\mathrm{SiO}_{2}$ interface vs porosity. Note that values above $30 \%$ porosity start to become unphysical. This is because pores eventually start to overlap, and water will go into the pores at high porosity. The curves correspond to Lorentz-Lorenz air (red curve), VAT air (black curve), VAT water (green curve), and LorentzLorenz water (blue curve) dielectric models.

increases, attraction turns to repulsion for $\mathrm{CO}_{2}$ molecules near the $\mathrm{SiO}_{2}$ surface. This is exactly the opposite as for binding of gas bubbles. We consider for comparison the VAT and Lorentz-Lorenz models and study both air and water filled pores.

\section{CONCLUSIONS}

To conclude, we have considered a simple model system with porous $\mathrm{SiO}_{2}$ in water as an adsorber of bubbles. It seems possible to enhance the presence of bubbles near the liquid-solid interface by changing the porosity. In contrast, dissolved $\mathrm{CO}_{2}$ molecules prefer to adsorb in regions where there is low porosity, potentially reducing their chance to be stored. In general, some fraction of the pores can be occupied by water, which we have also studied with simple model calculations.

\section{ACKNOWLEDGMENTS}

M.D. thanks the China Scholarship Council for support. P.T. gratefully acknowledges support from the European Commission. M.B., O.I.M., and C.P. acknowledge support from the Research Council of Norway (Project No. 221469). C.P. acknowledges support from the Swedish Research Council (Contract No. C0485101). We acknowledge access to HPC resources at NSC through SNIC/SNAC and at USIT through NORTUR. A $b$ initio calculations were undertaken at the NCI National Facility in Canberra, Australia, which is supported by the Australian Commonwealth Government.
[1] H. B. G. Casimir, Proc. K. Ned. Akad. Wet. 51, 793 (1948).

[2] S. K. Lamoreaux, Phys. Rev. Lett. 78, 5 (1997); 81, 5475 (1998).

[3] I. E. Dzyaloshinskii, E. M. Lifshitz, and P. P. Pitaevskii, Adv. Phys. 10, 165 (1961).

[4] P. Richmond and B. W. Ninham, Solid State Commun. 9, 1045 (1971).

[5] P. Richmond, B. W. Ninham, and R. H. Ottewill, J. Colloid Interface Sci. 45, 69 (1973).

[6] J. N. Munday, F. Capasso, and V. A. Parsegian, Nature (London) 457, 170 (2009).

[7] M. Dou, F. Lou, M. Boström, I. Brevik, and C. Persson, Phys. Rev. B 89, 201407(R) (2014).

[8] P. J. van Zwol and G. Palasantzas, Phys. Rev. A 81, 062502 (2010).

[9] A. D. Phan and N. A. Viet, Phys. Rev. A 84, 062503 (2011).

[10] M. Boström, B. E. Sernelius, I. Brevik, and B. W. Ninham, Phys. Rev. A 85, 010701(R) (2012).

[11] C. H. Anderson and E. S. Sabisky, Phys. Rev. Lett. 24, 1049 (1970).

[12] F. Hauxwell and R. H. Ottewill, J. Colloid Interface Sci. 34, 473 (1970).

[13] B. W. Ninham and V. A. Parsegian, Biophys. J. 10, 647 (1970).

[14] K. A. Milton, The Casimir Effect: Physical Manifestations of Zero-Point Energy (World Scientific, Singapore, 2001).

[15] V. A. Parsegian, van der Waals Forces: A Handbook for Biologists, Chemists, Engineers, and Physicists (Cambridge University Press, New York, 2006).

[16] B. W. Ninham and P. Lo Nostro, Molecular Forces and Self Assembly: In Colloid, Nano Sciences and Biology (Cambridge University Press, Cambridge, UK, 2010).
[17] J. Mahanty and B. W. Ninham, Dispersion Forces (Academic, London, 1976).

[18] A. Milling, P. Mulvaney, and I. Larson, J. Colloid Interface Sci. 180, 460 (1996).

[19] S. Lee and W. M. Sigmund, J. Colloid Interface Sci. 243, 365 (2001).

[20] A. A. Feiler, L. Bergstrom, and M. W. Rutland, Langmuir 24, 2274 (2008).

[21] C. T. Kresge, M. E. Leonowicz, W. J. Roth, J. C. Vartuli, and J. S. Beck, Nature (London) 359, 710 (1992).

[22] D. Zhao, J. Feng, Q. Huo, N. Melosh, G. H. Fredrickson, B. F. Chmelka, and G. D. Stucky, Science 279, 548 (1998).

[23] P. J. van Zwol, V. B. Svetovoy, and G. Palasantzas, in Casimir Physics, edited by D. Dalvit, P. Milonni, D. Roberts, and F. da Rosa, Lecture Notes in Physics Vol. 834 (Springer, Berlin, 2011), Chap. 10.

[24] W. Broer, G. Palasantzas, J. Knoester, and V. B. Svetovoy, Europhys. Lett. 95, 30001 (2011).

[25] W. Broer, G. Palasantzas, J. Knoester, and V. B. Svetovoy, Phys. Rev. B 85, 155410 (2012).

[26] R. F. Tabor, R. Manica, D. Y. C. Chan, F. Grieser, and R. R. Dagastine, Phys. Rev. Lett. 106, 064501 (2011).

[27] D.-M. Wang, Y.-M. Xu, D.-M. He, J. Guan, and O.-M. Zhang, Asia-Pac. J. Chem. Eng. 4, 691 (2009).

[28] G. E. King, SPE Annual Technical Conference and Exhibition, 19-22 September, Florence, Italy (Society of Petroleum Engineers, 2010).

[29] J. B. Curtiss, AAPG Bull. 86, 1921 (2002).

[30] A. J. Luhmann, X.-Z. Kong, B. M. Tutolo, K. Ding, M. O. Saar, and W. E. Seyfried, Environ. Sci. Technol. 47, 242 (2013). 
[31] R. Kumar, V. Bansal, R. M. Badhe, I. S. S. Madhira, V. Sugumaran, S. Ahmed, J. Christopher, M. B. Patel, and B. Basu, Fuel 113, 610 (2013).

[32] D. Tuschel, Spectroscopy 28, 3 (2013).

[33] F. M. Orr, Jr., Science 325, 1656 (2009).

[34] S. Taku Ide, K. Jessen, and F. M. Orr, Jr., Int. J. Greenhouse Gas Control 1, 481 (2007).

[35] Bo E. Sernelius, Surface Modes in Physics (Wiley-VCH, Berlin, 2001).

[36] M. R. Querry, D. M. Wieliczka, and D. J. Segelstein, Handbook of Optical Constants of Solids II (Academic, New York, 1991), p. 1059.

[37] M. Boström, B. E. Sernelius, and C. Persson, Eur. Phys. J. B 86, 43 (2013).
[38] M. Shishkin and G. Kresse, Phys. Rev. B 75, 235102 (2007).

[39] M. Dou and C. Persson, Phys. Status Solidi A 209, 75 (2012).

[40] M. M. Braun and L. Pilon, Thin Solid Films 496, 505 (2006).

[41] A. Navid and L. Pilon, Thin Solid Films 516, 4159 (2008).

[42] S. Bosch, J. Ferré-Borrull, N. Leinfellner, and A. Canillas, Surf. Sci. 453, 9 (2000).

[43] B. V. Derjaguin and I. I. Abrikossova, Sov. Phys.-Dokl. 1, 280 (1956).

[44] P. Thiyam, C. Persson, B. E. Sernelius, D. F. Parsons, A. MaltheSørenssen, and M. Boström, Phys. Rev. E 90, 032122 (2014).

[45] D. F. Parsons and B. W. Ninham, J. Phys. Chem. A 113, 1141 (2009).

[46] D. F. Parsons and B. W. Ninham, Langmuir 26, 1816 (2010). 